\title{
THE SCHNIRELMANN DENSITY OF THE SUMS OF THREE SQUARES
}

\author{
SAMUEL S. WAGSTAFF, JR.
}

ABSTRACT. The number in the title is $5 / 6$, which is the same as the asymptotic density of the set. The best possible upper and lower bounds (of the form $5 x / 6+A+B \log x$ ) are obtained for the number of positive integers less than $x$ which are a sum of three squares.

For $x>0$ let $S(x)$ and $N(x)$ be the numbers of positive integers not greater than $x$ which are and are not a sum of three squares of integers, respectively. It is well known that $N(x)$ is the number of numbers $\leq x$ which are of the form $4^{a}(8 b+7)$ where $a$ and $b$ are nonnegative integers. Since there are $[(1+x) / 8]$ integers $k \leq x$ with $k \equiv 7(\bmod 8)$, it is easy to see that

$$
N(x)=\sum_{j=0}^{\infty}\left[\frac{1+x 4^{-j}}{8}\right] .
$$

Using $x-1<[x] \leq x$ and the fact that the infinite sum terminates at $j_{0}=$ $\left[\log _{4}(x / 7)\right]$, one obtains the estimates $[1$, p. 250]

$$
\frac{x}{6}-\frac{7 \log x}{8 \log 4}-1<N(x)<\frac{x}{6}+\frac{\log x}{8 \log 4},
$$

which Landau [2] used to prove that the asymptotic density of the set of sums of three squares is $\lim _{x \rightarrow \infty} S(x) / x=5 / 6$. It is possible a priori that either $S(x) \geq 5 x / 6$ for all $x$ in which case the Schnirelmann density of this set is inf $_{x} S(x) / x=5 / 6$ or $S(x)<5 x / 6$ for some $x$ in which case the Schnirelmann density is less than $5 / 6$. The latter situation occurs for the set of squarefree numbers [4], which has asymptotic density $6 / \pi^{2}$ but Schnirelmann density 53/39. (The infimum is attained at $x=176$.) We will show that for all positive integers $x$ we have $S(x) \geq(5 x+1) / 6$, so that the first case applies for sums of three squares.

Theorem 1.

$$
N(x) \leq(x-1) / 6 \text { for } x \geq 1,
$$

and equality holds if and only if $x=2^{n}-1$ for some odd positive integer $n$.

Proof. Clearly the relative maxima of $N(x)-x / 6$ are at $x$ of the form $x=4^{a}(8 b+7)$. For such $x$ with $a \geq 2$ we have

Received by the editors April 17, 1974 and, in revised form, August 19, 1974. AMS (MOS) subject classifications (1970). Primary 10L 10; Secondary 10J05. Key words and phrases. Schnirelmann density, density, sums of three squares. 


$$
\begin{aligned}
& N(x)=\sum_{j=0}^{a-1}\left[\frac{4^{a-j}(8 b+7)}{8}\right]+\sum_{j=a}^{\infty}\left[\frac{1+4^{a-j}(8 b+7)}{8}\right] \\
&=\sum_{j=0}^{a-2} \frac{4^{a-j}(8 b+7)}{8}+\left[\frac{4(8 b+7)}{8}\right]+\sum_{j=0}^{\infty}\left[\frac{1+4^{-j}(8 b+7)}{8}\right] \\
&=\frac{4^{a}(8 b+7)}{8} \sum_{j=0}^{a-2} 4^{-j}+\left[4 b+\frac{7}{2}\right]+N(8 b+7) \\
&=\frac{4^{a}(8 b+7)}{8} \frac{4}{3}\left(1-4^{1-a}\right)+4 b+3+N(8 b+7) . \\
& N(x)=x / 6-4 b / 3-5 / 3+N(8 b+7) .
\end{aligned}
$$

A similar but simpler calculation shows that (1) holds also when $a=1$.

We now prove by induction on $b$ that

$$
N(8 b+7) \leq(8 b+7) / 6-1 / 6,
$$

and for all positive integers $a$ that

$$
N\left(4^{a}(8 b+7)\right) \leq(1 / 6) 4^{a}(8 b+7)-2 / 3 .
$$

For fixed $b$ (3) follows from (1) and (2):

$$
\begin{aligned}
N\left(4^{a}(8 b+7)\right) & =\frac{1}{6} 4^{a}(8 b+7)-\frac{4}{3} b-\frac{5}{3}+N(8 b+7) \\
& \leq \frac{1}{6} 4^{a}(8 b+7)-\frac{4}{3} b-\frac{5}{3}+\frac{8 b+7}{6}-\frac{1}{6} \\
& =\frac{1}{6} 4^{a}(8 b+7)-\frac{2}{3} .
\end{aligned}
$$

If $b=0$, then $N(8 b+7)=1=(8 b+7) / 6-1 / 6$. Let $b \geq 1, x=8 b+7$, and $y$ be the largest integer $<x$ which is not a sum of three squares. Then $y=x$ $-8, x-7$, or $x-3$. If $y=x-8$, then $N(y) \leq y / 6-1 / 6$, and

$$
\begin{aligned}
N(x) & =N(y)+1 \leq y / 6-1 / 6+1=(x-8) / 6+5 / 6 \\
& =x / 6-3 / 6<x / 6-1 / 6 .
\end{aligned}
$$

If $y=x-7$, then $N(y) \leq y / 6-2 / 3$, so

$$
\begin{aligned}
N(x) & =N(y)+1 \leq y / 6-2 / 3+1=(x-7) / 6+1 / 3 \\
& =x / 6-5 / 6<x / 6-1 / 6 .
\end{aligned}
$$

If $y=x-3$, then $N(y) \leq y / 6-2 / 3$, and

$$
N(x)=N(y)+1 \leq y / 6-2 / 3+1=(x-3) / 6+1 / 3=x / 6-1 / 6 .
$$

These inequalities show that $N(x) \leq(x-1) / 6$ for all $x \geq 1$ and that if equality holds, then $x=1$ or $x=7$ or for some positive integer $b$ we have $x=8 b+7$ and $8 b+4$ is not a sum of three squares. In the latter case the odd number $y=2 b+1$ cannot be a sum of three squares, whence $y=8 b^{\prime}+7$. 
Then $x=4 y+3$. Using (1) with $a=1$ we find

$$
\begin{aligned}
(x-1) / 6-1 & =N(x)-1=N(x-3)=N(4 y) \\
& =(x-3) / 6-4 b^{\prime} / 3-5 / 3+N(y)
\end{aligned}
$$

so that $N(y)=\left(8 b^{\prime}+7\right) / 6-1 / 6=(y-1) / 6$. If $b^{\prime}$ is a positive integer, we can repeat the above steps with $x$ replaced by $y$. Continuing this process we must eventually reach $y=7$. Since $N(y)=(y-1) / 6$ implies $N(4 y+3)=$ $(4 y+3-1) / 6$ by (1) again, we see that $N(x)=(x-1) / 6$ if and only if $x$ is one of the numbers $a_{n}$, where $a_{0}=1$ and $a_{n+1}=4 a_{n}+3$. An easy induction shows that $a_{n}=2^{2 n+1}-1$, and the theorem is proved.

Corollary. For every positive integer $x$ we have $S(x) \geq(5 x+1) / 6$.

Proof. $S(x)=x-N(x)$ for every positive integer $x$.

Corollary. The Schnirelmann density of the set of all sums of three squares of integers is $5 / 6$.

We have reduced Landau's upper bound on $N(x)$ by about $(1 / 8) \log _{4} x$. The next theorem narrows his lower bound on $N(x)$ by about $(5 / 24) \log _{4} x$.

Theorem 2. For all integers $x \geq 2$ we have

$$
N(x) \geq x / 6-(1 / 3)\left(1+2 \log _{4}(1+(3 / 4)(x-2))\right),
$$

and equality holds if and only if $x$ is of the form $x=4\left(4^{n}-1\right) / 3+2$, where $n$ is a nonnegative integer.

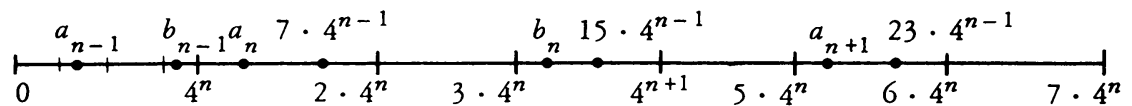

Proof. For each nonnegative integer $n$ define positive integers $a_{n}$ and $b_{n}$ by $a_{n}=4\left(4^{n}-1\right) / 3+2, b_{n}=10\left(4^{n}-1\right) / 3+4$. Note that $a_{0}=2, b_{0}=4$, $a_{n+1}=b_{n}+2 \cdot 4^{n}$, and $b_{n}=a_{n}+2 \cdot 4^{n}$ for each nonnegative integer $n$, and that $a_{n}=a_{n-1}+4^{n}, a_{n} \equiv b_{n} \equiv 6(\bmod 8)$, and

$$
4^{n}<a_{n}<7 \cdot 4^{n-1}<3 \cdot 4^{n}<b_{n}<15 \cdot 4^{n-1}
$$

for every positive integer $n$. Let $f(x)=x / 6-N(x)$. We will show first by induction on $n$ that $f\left(a_{n}\right)=(2 n+1) / 3$ and $f\left(b_{n}\right)=(2 n+2) / 3$ for every nonnegative integer $n$. These equations are easily verified for small $n$, so we may a ssume $n \geq 2$. Every $a_{n}$ and $b_{n}$ is a sum of three squares. Let \#A denote the cardinality of the set $A$. Since $a_{n}<7 \cdot 4^{n-1}$, every number $k$ between $a_{n-1}$ and $a_{n}$ which is not a sum of three squares is of the form $k=$ $4^{a}(8 b+7)$ with $a \leq n-2$, and

$$
\begin{aligned}
& \#\left\{a_{n-1}<k \leq a_{n} \mid k \text { is not a sum of three squares }\right\} \\
& =\#\left\{0<k \leq 4^{n} \mid k \text { is not a sum of three squares }\right\} \\
& =4^{n}\left(\frac{1}{8}+\frac{1}{8 \cdot 4}+\frac{1}{8 \cdot 16}+\cdots+\frac{1}{8 \cdot 4^{n-2}}\right)=\frac{4^{n}-4}{6} .
\end{aligned}
$$


Hence $N\left(a_{n}\right)=N\left(a_{n-1}\right)+\left(4^{n}-4\right) / 6$. Assume by induction that $f\left(a_{n-1}\right)=$ $(2 n-1) / 3$. Then

$$
f\left(a_{n}\right)=\frac{a_{n}}{6}-N\left(a_{n}\right)=\frac{a_{n}}{6}-N\left(a_{n-1}\right)-\frac{4^{n}}{6}+\frac{4}{6}=f\left(a_{n-1}\right)+\frac{2}{3}=\frac{2 n+1}{3},
$$

which is the first assertion. Every number $k$ between $a_{n}$ and $b_{n}$ which is not a sum of three squares is of the form $k=4 a(8 b+7)$ with $a \leq n-1$. Hence $\#\left\{a_{n}<k \leq b_{n} \mid k\right.$ is not a sum of three squares $\}$

$$
\begin{aligned}
& =\#\left\{0<k \leq 2 \cdot 4^{n} \mid k \text { is not a sum of three squares }\right\} \\
& =1+2 \cdot\left(4^{n}-4\right) / 6 .
\end{aligned}
$$

Therefore $N\left(b_{n}\right)=N\left(a_{n}\right)+1+\left(4^{n}-4\right) / 3$. We have already proved that $f\left(a_{n}\right)$ $=(2 n+1) / 3$, so that

$$
f\left(b_{n}\right)=\frac{b_{n}}{6}-N\left(b_{n}\right)=N\left(a_{n}\right)-\frac{2 \cdot 4^{n}}{6}-1+\frac{4}{3}=f\left(a_{n}\right)+\frac{1}{3}=\frac{2 n+2}{3} .
$$

Notice that $f\left(b_{n}\right)=f\left(a_{n}\right)+1 / 3$ and $f\left(a_{n+1}\right)=f\left(b_{n}\right)+1 / 3$. Also since every $a_{n}$ and $b_{n}$ is a sum of three squares we have

$$
f\left(a_{n}-1\right)=\frac{2 n+1}{3}-\frac{1}{6} \text { and } f\left(b_{n}-1\right)=\frac{2 n+2}{3}-\frac{1}{6} .
$$

Next we will prove that

$$
x<b_{n}-1 \text { implies } f(x) \leq f\left(a_{n}\right)
$$

and

$$
x<a_{n+1}-1 \text { implies } f(x) \leq f\left(b_{n}\right) .
$$

We use induction on $n$ and may assume $n \geq 2$. Suppose $x<b_{n}-1$. Then $x-2 \cdot 4^{n}<a_{n}-1$. Let $y=x-t 4^{n}(t=0,1$, or 2$)$ so that $0<a_{n}-4^{n}-1$ $\leq y<a_{n}-1$. We are assuming that $f(y) \leq f\left(b_{n-1}\right)$. We have $f(x)=f(y)+$ $t 4^{n} / 6-m$, where

$m=\#\{y<k \leq x \mid k$ is not a sum of three squares $\}=t\left(4^{n}-4\right) / 6+z$, where

$$
z= \begin{cases}1 & \text { if } x \geq 7 \cdot 4^{n-1} \\ 0 & \text { if } x<7 \cdot 4^{n-1}\end{cases}
$$

Hence

$$
f(x)=f(y)+2 t / 3-z \leq f\left(b_{n-1}\right)+2 t / 3-z=f\left(a_{n}\right)+(2 t-1) / 3-z .
$$

Now $z \geq 0$, so if $t=0$, we have proved (4). Otherwise $t=1$ or 2 so $x \geq a_{n}$ $+4^{n}>7 \cdot 4^{n-1}$. Then $z$ must be 1 and we have $f(x) \leq f\left(a_{n}\right)+(2 t-1) / 3-1$ $\leq f\left(a_{n}\right)$. This proves (4).

Now suppose $x<a_{n+1}-1$. If $x \leq b_{n}$, we are done by the above. If $x>b_{n}$, let $y=x-2 \cdot 4^{n}$. Then $a_{n} \leq y<b_{n}-1$, so $f(y) \leq f\left(a_{n}\right)$ by induction, and $f(x)=f(y)+2 \cdot 4^{n} / 6-m$, where 
$m=\#\{y<k \leq x \mid k$ is not a sum of three squares $\}=2\left(4^{n}-4\right) / 6+1$, since exactly one of the numbers $7 \cdot 4^{n-1}, 15 \cdot 4^{n-1}$ is in the set. Therefore which is (5).

$$
f(x)=f(y)+8 / 6-1 \leq f\left(a_{n}\right)+1 / 3=f\left(b_{n}\right),
$$

We now know that $f(x) \leq g(x)$ for each positive integer $x$, where

$$
g(x)=\left\{\begin{array}{lll}
(2 n+1) / 3 & \text { if } & a_{n} \leq x<b_{n}-1 \\
(4 n+3) / 6 & \text { if } & x=b_{n}-1 \\
(2 n+2) / 3 & \text { if } & b_{n} \leq x<a_{n+1}-1 \\
(4 n+5) / 6 & \text { if } & x=a_{n+1}-1
\end{array}\right.
$$

Let $h(x)=(1 / 3)\left(1+2 \log _{4}(1+(3 / 4)(x-2))\right)$. Then $h\left(a_{n}\right)=g\left(a_{n}\right)=f\left(a_{n}\right)$ $(n \geq 0)$. To prove the theorem, it suffices to show that $h(x)>g(x)$ when $x \geq 2$ and $x$ is not one of the $a_{n}$. For $x=a_{n}-1$ we have $g(x)=(4 n+1) / 6$ and

$$
h(x)=\frac{1}{3}\left(1+2 n+2 \log _{4}\left(1-3 / 4^{n+1}\right)\right)=g(x)+\frac{1}{6}+\frac{2}{3} \log _{4}\left(1-3 / 4^{n+1}\right) .
$$

But $1+4 \log _{4}(1-3 / s)>0$ for $s>6+3 \sqrt{2}$ so that $h\left(a_{n}-1\right)>g\left(a_{n}-1\right)$ for positive integers $n$. (However for $n=0$ we have $h(1)<g(1)$.) Now $b_{n}=$ $\left(a_{n}+a_{n+1}\right) / 2$. Since $\log _{4}$ is concave down we have

$$
b\left(b_{n}\right)>\frac{1}{2}\left(b\left(a_{n}\right)+b\left(a_{n+1}\right)\right)=\frac{1}{2}\left(g\left(a_{n}\right)+g\left(a_{n+1}\right)\right)=g\left(b_{n}\right) .
$$

Finally $b_{n}-1=\left(\left(a_{n}-1\right)+\left(a_{n+1}-1\right)\right) / 2$, whence $b\left(b_{n}-1\right)>g\left(b_{n}-1\right)$, and the proof is complete.

Note that the bounds on $f(x)$ given by Theorems 1 and 2 are the best possible of the form $A+B \log x$.

Corollary. For all integers $x \geq 2$ we have

$$
\frac{5}{6} x+\frac{1}{6} \leq S(x) \leq \frac{5}{6} x+\frac{1}{3}\left(1+2 \log _{4}\left(1+\frac{3}{4}(x-2)\right)\right) \text {. }
$$

The set $E$ of all positive integers which are not a sum of three squares has density $1 / 6$ but Schnirelmann density 0 because 1 is not in $E$. One might hope that if we adjoin some finite set of integers (including 1 ) to $E$, we would get a set with Schnirelmann density $1 / 6$. An easy calculation shows that the Schnirelmann density of $E \cup\{1\}$ is $3 / 22$ and that of $E \cup\{1,2\}$ is $7 / 43$, both less than 1/6. Indeed, if $F$ is any finite set, then $E \cup F$ has Schnirelmann density $<1 / 6$ because $h(x)$ is not bounded above.

The quadratic form $x^{2}+y^{2}+2 z^{2}$ represents all positive integers not of the form $4^{a}(16 b+14)=2 \cdot 4^{a}(8 b+7)$. Let $S_{1}(x)$ and $N_{1}(x)$ be the number of positive integers not exceeding $x$ which are and are not represented by this 
quadratic form. Then $N_{1}(x)=N(x / 2)$ Theorem 1 gives $N_{1}(x) \leq(x-2) / 12$ for $x \geq 2$, where equality holds if and only if $x=2^{n}-2$ for some even positive integer $n$. Therefore

$$
S_{1}(x)=x-N_{1}(x) \geq(11 x+2) / 12 \quad(x \geq 2),
$$

so the Schnirelmann density of the set of integers represented by $x^{2}+y^{2}+$ $2 z^{2}$ is $11 / 12$, which is the same as the asymptotic density of the set.

It is well known [3] that $x^{2}+2 y^{2}+2 z^{2}$ and $x^{2}+2 y^{2}+4 z^{2}$ represent the same integers as $x^{2}+y^{2}+z^{2}$ and $x^{2}+y^{2}+2 z^{2}$, respectively, so we have analogous theorems for the Schnirelmann and asymptotic densities of the sets of integers represented by these quadratic forms.

However, it is not always the case that if a positive definite ternary quadratic form represents 1 , then the Schnirelmann density of the set of integers it represents is the same as the asymptotic density. For example, let $S_{2}(x)$ and $N_{2}(x)$ be the numbers of positive integers $\leq x$ which are and are not represented by the form $x^{2}+y^{2}+3 z^{2}$. This form represents all positive integers not of the form $9^{a}(9 b+6)$. (See [3].) Hence

$$
N_{2}(x)=\sum_{j=0}^{\infty}\left[\frac{3+x 9^{-j}}{9}\right] \text {, }
$$

and this sum actually terminates at $j_{0}=\left[\log _{9}(x / 6)\right]$. Let $f(n)=n / 8-N_{2}(n)$. The methods of Theorems 1 and 2 show that the local extrema of $f(n)$ are

and

$$
f\left(3\left(9^{n}-1\right) / 4\right)=-n / 4 \quad(n \geq 0)
$$

$$
f\left(5\left(9^{n}-1\right) / 8\right)=5 n / 8 \quad(n \geq 0),
$$

and lead to these upper and lower bounds on $N_{2}(x)$ :

Theorem 3. For every nonnegative integer $x$ we have

$$
\frac{x}{8}-\frac{5}{8} \log _{9}\left(1+\frac{8 x}{5}\right) \leq N_{2}(x) \leq \frac{x}{8}+\frac{1}{4} \log _{9}\left(1+\frac{4 x}{3}\right),
$$

where equality holds on the left if and only if $x$ is of the form $x=5\left(9^{n}-1\right) / 8$, and equality holds on the right if and only if $x$ is of the form $x=3\left(9^{n}-1\right) / 4$.

Here again the bounds on $f(x)$ are the best possible of the form $A+$ $B \log x$.

The resultant inequalities on $S_{2}(x)$ make it easy to prove that the Schnirelmann density of the set of integers represented by the form $x^{2}+y^{2}+$ $3 z^{2}$ is $5 / 6$ (infimum attained at $x=6$ ), while the asymptotic density of the set is $7 / 3$.

The author thanks Professor P. T. Bateman for suggesting the problem and for some helpful comments on the first draft of this paper. The referee pointed out that there is an interesting application of $S(x) \sim 5 x / 6$ to thermal 
radiation in finite cavities given on p. 521 of H. P. Baltes and F. K. Kneubühl, Helv. Phys. Acta. 45 (1972), 481-529.

\section{REFERENCES}

1. E. Landau, Elementare Zahlentheorie, Teubner, Leipzig, 1927; English transl., Elementary number theory, Chelsea, New York, 1958. MR 19, 1159.

2. - Über die Einteilung der positiven ganzen Zahlen in vier Klassen nach der Mindestzahl der zu ihrer additiven Zusammensetzung erforderlichen Quadrate, Arch. Math. Phys. 13 (1908), 304-312.

3. S. Ramanujan, On the expression of a number in the form $a x^{2}+b y^{2}+c z^{2}+$ $d u^{2}$, Proc. Cambridge Philos. Soc. 19 (1917), 11-21.

4. Kenneth Rogers, The Schnirelmann density of the squarefree integers, Proc. Amer. Math. Soc. 15 (1964), 515-516. MR 29 \#1192.

DEPARTMENT OF MATHEMATICS, UNIVERSITY OF ILLINOIS, URBANA, ILLINOIS 61801 